\title{
Una Sonrisa en la Boca Hace que los Ojos Parezcan Alegres
}

\section{A Smile Makes the Eyes Look Happy}

\author{
Andrés Fernández Martín, Pedro Avero y Manuel Gutiérrez Calvo \\ Universidad de La Laguna, España.
}

Disponible online 30 de abril de 2012

\begin{abstract}
El presente estudio investiga la influencia de la sonrisa sobre la expresión de los ojos, sean éstos alegres o no, en comparación con la influencia de una boca triste o de enfado. La muestra final estuvo compuesta por treinta y seis estudiantes de primer curso del Grado de Psicología. Los participantes miraban inicialmente a la boca y luego juzgaban si la expresión de los ojos era alegre (o triste o de enfado) o no. La expresión de ojos y boca podía ser congruente (v.g., ojos y boca alegres) o incongruente (v.g., ojos de miedo/boca alegre), o bien la cara tenía los ojos visibles pero la boca enmascarada (condición de control). Los resultados indicaron que, en condiciones de congruencia, las expresiones de alegría, tristeza y enfado en la región de la boca facilitaban de modo similar la identificación correcta de la expresión de los ojos. En cambio, en condiciones de incongruencia, la boca sonriente produjo mayores efectos de interferencia que las de tristeza o enfado: La sonrisa indujo a evaluar erróneamente los ojos no alegres como alegres e incrementó el tiempo para su identificación correcta. Los efectos de facilitación son explicados por un mecanismo de priming guiado por la primera mirada. La elevada saliencia perceptiva y el alto valor diagnóstico de la sonrisa explican los efectos de interferencia.
\end{abstract}

Palabras clave: Expresión Facial; Emoción; Sonrisa; Reconocimiento.

We investigated how smiles influence perceptions of what the eyes are expressing, whether happy or sad, compared to the influence of sad or angry mouths. Participants were thirty-six first year Psychology students. Participants initially looked at the mouth and then judged whether the eye expression was happy, sad or angry or not. The expression of the eyes and mouth could be congruent (eg, both happy) or incongruent (eg, fearful eyes, smiling mouth); the eyes were visible but the mouth was masked in the control condition. The results suggest that, under eye-mouth congruence conditions, looking at the smiling, sad and angry mouths similarly facilitated the correct identification of the eyes. In contrast, under incongruence conditions, a smiling mouth led to greater interference than sad or angry mouths; that is, the smile misled the viewers into judging non-happy eyes as happy, and increased the time needed to correctly identify their expression. The facilitation effects are explained by a priming mechanism guided by the first gaze directed at the mouth. The interference effects are explained as a function of the high visual saliency and diagnostic value of the smile.

Keywords: Facial Expression; Emotion; Smile; Recognition.

Correspondencia: Andrés Fernández Martín. Departamento de Psicología Cognitiva, Social y Organizacional. Universidad de La Laguna. 38205 Tenerife, España. Phone: +34 922317514 Fax:+34922317 461. E-mail: afdezm@ull.es. E-mail de los otros autores: Pedro Avero: paverod@ull. es, Manuel Gutiérrez Calvo: mgcalvo@ull.es

La investigación referida en este artículo ha sido subvencionada por el Ministerio de Ciencia e Innovación al Proyecto de Investigación PSI200907245, así como por la Agencia Canaria de Investigación, Innovación y Sociedad de la Información y los Fondos FEDER al Proyecto NEUROCOG. 
La sonrisa es un gesto facial muy frecuente e influyente en las relaciones sociales (Johnston, Miles, y MaCrae, 2010; Krumhuber y Manstead, 2009). Aunque generalmente se emplea para expresar alegría, también es cierto que a menudo, cuando va acompañada de ojos no alegres, trasmite mera cortesía o incluso esconde sentimientos menos positivos (nerviosismo, dominancia, etc.) (Ambadar, Cohn y Reed, 2009; Niedenthal et al., 2010). La región de la boca y la de los ojos en una cara son las principales fuentes de información expresiva. La sonrisa genuina conlleva cambios en el área de la boca (v.g., comisuras de los labios estiradas hacia atrás y hacia arriba) y otros algo menos evidentes en la zona de los ojos (el denominado marcador Duchenne; v.g., bolsas y arrugas debajo y alrededor de ellos; Ekman y Friesen, 1978). Si bien la presencia de los cambios en el área de los ojos no garantiza que la sonrisa sea espontánea, su ausencia indica que la sonrisa es forzada intencionadamente y, probablemente, no genuina (Krumhuber y Manstead, 2009).

El objetivo del presente estudio es, primero, determinar si una boca sonriente en el emisor induce al observador a percibir la expresión de los ojos como de alegría, tanto si ésta es realmente alegre como si no lo es. En segundo lugar, investigamos si la posible influencia de la boca sobre los ojos es un fenómeno específico de las caras con sonrisa, o si ocurre también con otras expresiones faciales. Estos objetivos son importantes teóricamente, por cuanto sirven para establecer la existencia de un mecanismo de proyección desde una fuente expresiva dominante (v.g., la boca) sobre otras (v.g., los ojos), amplificando o reduciendo el poder informativo de éstas. El concepto de proyección de la boca sonriente hacia los ojos fue inicialmente sugerido por Kontsevich y Tyler (2004). El mecanismo de proyección implica que un rasgo expresivo de una región de la cara puede influir sobre otro debido al procesamiento configuracional e integrador de rasgos faciales individuales, que se produce de modo automático en la percepción de caras (Calder et al., 2000; Richler et al., 2009). Los objetivos de este estudio son también relevantes en términos prácticos. Dado que en el mundo social la sonrisa en la boca a menudo no va acompañada por una expresión de alegría en los ojos, cabe pensar si la boca sonriente distorsionará la percepción de los ojos, impidiendo así apreciar la verdadera expresión de éstos. Si así fuera, los

\footnotetext{
${ }^{1}$ Es preciso hacer una distinción entre los procesos de detección de caras en tareas de búsqueda visual y los de reconocimiento de expresiones faciales en tareas de categorización. En las tareas de búsqueda se trata, simplemente, de decidir si una cara es diferente de otras circundantes; en las de categorización, de identificar cuál es la expresión de una cara. En el presente artículo nos referimos a los procesos de reconocimiento. Sobre ellos existe consenso empírico en la ventaja de las caras con expresión de alegría (véanse referencias en el texto principal). Respecto a los procesos de detección, existe evidencia sobre la ventaja de las caras de enfado cuando se utilizan caras esquemáticas, pero cuando se presentan caras reales, es más frecuente la ventaja de
}

observadores podrían interpretar erróneamente una sonrisa no genuina y, en consecuencia, dirigir su conducta social de modo no adecuado hacia el emisor.

La investigación previa sobre el reconocimiento de expresiones faciales emocionales ha demostrado consistentemente una ventaja de las caras de alegría. Éstas se reconocen mejor y más rápidamente que cualquiera de las otras expresiones emocionales básicas (enfado, tristeza, miedo, asco, y sorpresa; Calvo y Lundqvist, 2008; Juth et al., 2005; Leppänen y Hietanen, 2004; Leppänen, Tenhunen y Hietanen, 2003; Loughead et al., 2008; Milders, Sahraie y Logan, 2008; Palermo y Coltheart, 2004; Tottenham et al., 2009). ${ }^{1}$ Esta ventaja se ha atribuido, precisamente, a la sonrisa. La presencia de una boca sonriente se ha revelado como el elemento decisivo a la hora de reconocer o categorizar las expresiones faciales emocionales (Bassili, 1979; Calder et al., 2000; Calvo y Marrero, 2009; Kohler et al., 2004; Nusseck et al., 2008; Smith et al., 2005). Por ejemplo, Calder et al. (2000) encontraron que las expresiones alegres fueron reconocidas a partir de la parte inferior de la cara (con la boca) con tanta precisión (sólo el 1\% de errores), e incluso más rápidamente, que cuando se presentaba la cara completa y, por supuesto, que cuando se presentaba sólo la mitad superior (con los ojos).

Dentro de la sonrisa hay dos características especiales que pueden explicar los efectos facilitadores de ésta sobre el reconocimiento de la expresión: la distintividad categorial y la saliencia perceptiva. La distintividad de un rasgo facial expresivo hace referencia al grado en que el rasgo es propio y exclusivo y, por tanto, diagnóstico de una categoría. En este sentido, la sonrisa es un rasgo muy distintivo de la expresión de alegría, mientras que otros rasgos faciales son más comunes a varias categorías expresivas $\mathrm{y}$, por tanto, menos distintivos (Calvo y Marrero, 2009; Kohler et al., 2004). ${ }^{2}$ Debido a ello, el rasgo individual de la boca sonriente puede ser utilizado por el observador como una señal clara y un atajo para el reconocimiento de una cara alegre, casi sin tener que procesar otras partes de ésta. En contraste, la identificación de otras expresiones exigiría un análisis combinado de varios rasgos faciales, debido a lo cual el proceso sería más lento y sujeto a errores. Ahora bien, basándose únicamente en la sonrisa, el observador corre el riesgo de equivocarse si la sonrisa va acompañada de unos ojos

las alegres (véase revisión crítica en Calvo y Nummenmaa, 2008, y Frischen, Eastwood, y Smilek, 2008).

${ }^{2}$ La distintividad y la saliencia de la sonrisa serían las causas directas de la ventaja en el reconocimiento de las caras alegres. No obstante, podemos considerar también las razones adaptativas de esa ventaja, como posibles causas indirectas. Entre esas razones adaptativas destaca la importante función de la sonrisa en el inicio y mantenimiento de lazos sociales (Tomkins, 1962). Tal ventaja contribuiría a maximizar la obtención de recompensas, así como el establecimiento de alianzas y colaboraciones con otras personas (Oatley y Jenkins, 1996). 
no alegres, pudiendo interpretar una expresión de alegría "fingida" como si fuese genuina. Por su parte, la saliencia perceptiva hace referencia al grado en que un rasgo expresivo destaca físicamente sobre otros circundantes, en términos de energía, contraste y orientación espacial (Itti y Koch, 2000; Torralba et al., 2006). Precisamente, mediante modelos computacionales de la saliencia visual, se ha encontrado que la boca sonriente no sólo es más saliente que cualquier otra región de las caras alegres, sino también más saliente que cualquier otra región del resto de expresiones (véase Calvo y Nummenmaa, 2008). Esto asegura que la sonrisa capte pronto y poderosamente la atención, garantizándose así su procesamiento temprano como elemento distintivo. Por esa razón cabe predecir que la sonrisa tenga un papel muy influyente en la percepción y valoración de la expresión facial.

Este análisis de la distintividad y la saliencia de la sonrisa es relevante para abordar los objetivos del presente estudio. ¿Es la boca sonriente tan saliente y distintiva que "deslumbra" al observador y le impide apreciar los matices expresivos de otras partes de la cara? ¿Es la boca sonriente tan saliente y distintiva que se "proyecta" sobre otras partes de la cara, tales como los ojos, haciéndolos parecer alegres aunque no lo sean? Si así fuera, una vez que hemos mirado a una boca sonriente, nuestra valoración del resto de la cara se vería "contaminado" por la impresión que la sonrisa nos deja. Esto implica que tendríamos dificultad para percibir e interpretar unos ojos no alegres (v.g., no expresivos, o incluso tristes, de miedo, etc.) como tales cuando aparecen en una configuración facial con una boca sonriente, en comparación con cuando los mismos ojos aparecen en una cara no alegre, o cuando la boca no es visible. En definitiva, esto nos lleva a predecir una dificultad para discriminar entre las sonrisas genuinas (con boca sonriente y ojos alegres) y las fingidas (con boca sonriente pero ojos no alegres).

Para investigar la influencia de la sonrisa, y su comparación con la de otras expresiones, en el presente estudio utilizamos tres tipos de estímulos: a) caras con expresiones genuinas de alegría, tristeza, enfado, miedo, asco, sorpresa, y caras neutras (no expresivas), en las cuales los ojos eran congruentes con la boca; b) caras con expresiones mixtas, empleando un paradigma de caras compuestas (composite face paradigm; Leppänen y Hietanen, 2007; Tanaka et al., in press), en las cuales la boca era sonriente, o de tristeza, o de enfado, mientras que los ojos correspondían a cada una de las otras expresiones básicas y, por tanto, había incongruencia entre ojos y boca; y c) pseudo-caras de control en las cuales la mitad inferior aparecía difuminada por una máscara que ocultaba la boca. Los participantes tenían que juzgar la expresión de los ojos. La cara iba precedida por una señal visual que dirigía la mirada inicial del observador a la boca, usando una técnica de señalización (pre-cueing; e.g., Eckstein, Shimozaki, y Abbey, 2002; Ortells et al., 2001). A continuación el sujeto tenía tiempo libre para decidir sobre la expresión de los ojos. Es importante resaltar que los ojos objeto de comparación (neutros, de enfado, miedo, etc.) eran los mismos en las tres condiciones (expresiones genuinas, mixtas, y de control).

Este paradigma posibilita tres tipos de comparaciones relevantes a nuestros objetivos. Primero, permite averiguar los posibles efectos de facilitación sobre la evaluación de la expresión de los ojos por parte de las bocas con expresión congruente con ellos, con respecto a cuando la boca está ausente (condición de control). Segundo, y más novedoso y específico para el presente planteamiento, permite indagar sobre los efectos de inhibición o interferencia cuando la expresión de la boca es incongruente con la de los ojos, respecto a cuando la boca está ausente (condición de control). Y, en tercer lugar, permite investigar si los efectos de facilitación y los de interferencia son mayores cuando la boca sonríe que cuando expresa enfado o tristeza.

\section{Participantes \\ Se ofreció créditos de prácticas a estudiantes de primer curso de psicología de la Universidad de La Laguna por su par- ticipación voluntaria en el experimento, como complemento formativo en una asignatura relacionada con los procesos emo- cionales. La muestra final estuvo compuesta por treinta y seis estudiantes (28 mujeres), de entre 18 y 25 años.}

\section{Estímulos}

Se presentaron 168 caras extraídas de la base KDEF (Karolinska Directed Emotional Faces; Lundqvist, Flykt y Öhman, 1998). Estas caras corresponden a los siguientes 24 modelos: 12 mujeres (KDEF no. 01, 02, 07, 11, 14, 19, 20, 22, 2629 , 31,35 ) y 12 hombres (KDEF no. 03, 05, 06, 10, 11, 12, 22, 23, $24,25,31,35)$. De cada modelo se utilizaron (a) las siguientes categorías con expresiones genuinas: alegría, tristeza, enfado, miedo, asco, sorpresa, y neutra. Además, construimos (b1) 144 caras con expresiones mixtas, 6 de cada modelo, que combinan la sonrisa de las caras alegres con los ojos del resto de las categorías; (b2) 144 caras con expresiones mixtas, 6 de cada modelo, que combinan una boca de enfado con los ojos del resto de las categorías; y (b3) 144 caras con expresiones mixtas, 6 de cada modelo, que combinan una boca triste con los ojos del resto de las categorías. Finalmente, (c) para la condición de control, sin boca, se formaron 168 óvalos que incluían la mitad superior -con los ojos- de las caras genuinas y la mitad inferior cubierta por una máscara de Fourier. Para generar las expresiones mixtas, fusionamos la mitad inferior (alegre, enfado, o triste) de la cara de cada modelo con la mitad superior (cada una de las otras seis expresiones genuinas) de la misma persona, mediante el software Adobe ${ }^{\circledR}$ Photoshop ${ }^{\circledR}$ CS3. La Figura 1 muestra un ejemplo de cada categoría genuina y mixta (ejemplos de las caras en la condición de control pueden verse en la Figura 2). 


\section{Figura 1}

Ejemplo de cada una de las categorías expresivas genuinas y de cada una de las expresiones mixtas utilizadas, combinando las bocas alegres, de enfado y tristeza con los ojos del resto de expresiones emocionales.

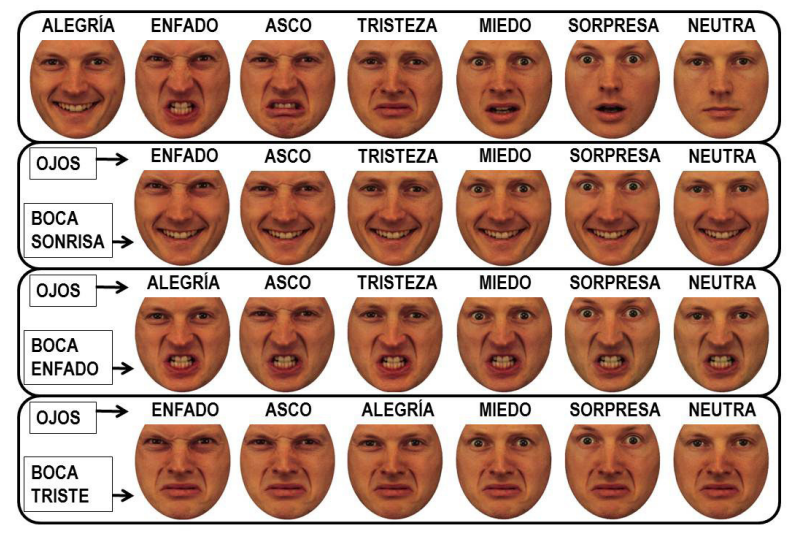

\section{Aparatos}

La presentación de las caras y el registro de las respuestas se llevaron a cabo mediante el programa E-Prime (versión 2.0). Las caras aparecían en un monitor de 17 " sobre un fondo en negro. Las dimensiones de las caras eran de 8.4 x 6.4 grados de ángulo visual a una distancia de $60 \mathrm{~cm}$ del estímulo respecto al observador. Estas medidas corresponden, aproximadamente, al tamaño de la cara real de una persona $(18.5 \mathrm{~cm}$ de alto $\times 13.8$ $\mathrm{cm}$ de ancho) vista a una distancia de 1 metro entre dos personas (véanse Henderson, Williams y Falk, 2005; Remland, Jones y Brinkman, 1995).

\section{Procedimiento y Medidas}

Al inicio de la sesión experimental se dijo a los participantes que verían caras con diferentes expresiones, precedidas por un pequeño rectángulo. Tenían que mirar primero al rectángulo y, al desaparecer éste, a la cara. Su tarea consistía en decidir y responder rápidamente si los ojos expresaban una determinada emoción o no. Al inicio de cada uno de tres bloques de 72 ensayos cada uno se indicó la expresión objetivo (target) que los participantes tenían que utilizar como criterio de evaluación y decisión. En un bloque, la expresión objetivo era la de alegría (es decir, los participantes tenían que juzgar si los ojos de cada una de las 72 caras expresaban alegría); en otro bloque, era la de enfado, y en otro, la de tristeza. Las caras en cada bloque eran diferentes para un mismo sujeto. El orden de los bloques (y la expresión objetivo) fue contrabalanceado.

La secuencia de eventos en cada ensayo fue como sigue: después de un intervalo de $750 \mathrm{~ms}$ respecto al final del ensayo anterior (contado a partir de la respuesta del sujeto), aparecía un rectángulo durante $250 \mathrm{~ms}$ como señal visual para guiar la atención inicialmente hacia la boca. Para ello, primero, dicha figura geométrica se presentaba abrupta y brevemente $(250 \mathrm{~ms})$ para capturar la mirada y, segundo, ocupaba espacialmente el lugar en el que a continuación aparecía la boca. Si bien los participantes disponían de tiempo libre para mirar a la cara una vez que ésta aparecía, era importante asegurar que miraban a la boca, a fin de establecer la influencia de ésta sobre la valoración posterior de los ojos. Al desaparecer la señal visual aparecía la cara, permaneciendo hasta que el participante respondía manualmente (presionando una de dos teclas) si los ojos se correspondían o no con la expresión objetivo predefinida. Una vez registrada la respuesta de los participantes, se calculó la probabilidad de responder SI o NO y los tiempos de reacción de los aciertos. Véase la Figura 2.

\section{Diseño}

Para determinar la influencia relativa de la boca sonriente, la de enfado, y la triste sobre los ojos, utilizamos dos tipos de planteamientos complementarios en el diseño experimental. El primero permite examinar la influencia facilitadora de la boca cuando la expresión de ésta es congruente con la de los ojos. Esto se aplica a las caras con expresiones genuinas. Para ello se combinó el tipo de expresión objetivo (alegre vs. enfado vs. triste) con el tipo de boca (congruente con la expresión de los ojos vs. ausencia de boca-condición de control), siendo ambos factores intra-sujeto. El segundo planteamiento permite explorar la influencia interferidora de la boca cuando es incongruente con la expresión de los ojos, lo cual se aplica a las caras con expresión mixta. Para esto combinamos el tipo de expresión objetivo (alegre vs. enfado vs. triste) con el tipo de boca (incongruente con la expresión de los ojos vs. ausencia de boca-condición de control), siendo ambos factores también intra-sujeto.

Para cada expresión objetivo (v.g., alegre) y bloque, cada participante recibió: (a) 24 ensayos en los cuales la boca era incongruente con los ojos (v.g., boca de enfado y ojos alegres); (b) 24 ensayos en los cuales los mismos ojos (v.g., alegres) aparecían en una cara con la boca enmascarada (control); y (c) 24 ensayos en los cuales (c1) la boca era congruente con los ojos (12 ensayos; v.g., boca con sonrisa y ojos alegres) o (c2) los mismos ojos aparecían en una cara con la boca enmascarada (control; 12 ensayos). Las comparaciones críticas tienen lugar entre (a) y (b), para averiguar los efectos "contaminantes" o de interferencia de la boca sobre los ojos; las comparaciones entre (c1) y (c2) sirven para explorar los efectos de facilitación.

Es preciso resaltar que el tipo de expresión de los ojos era diferente en los dos planteamientos metodológicos descritos: mientras que en el relativo a los efectos de incongruencia los ojos nunca coincidían con la expresión objetivo (y, por tanto, la respuesta correcta era siempre "No"), en el correspondiente a los efectos de congruencia los ojos siempre coincidían con la expresión objetivo (y, por tanto, la respuesta correcta era siempre "Sí"). Las expresiones de los ojos en la condición de control, con boca enmascarada, eran diferentes en uno y otro planteamiento y, por consiguiente, no eran directamente comparables. El punto importante, no obstante, es que, dentro de cada planteamiento, las expresiones de los ojos eran idénticas en la condición de control y en la experimental (ya congruente, ya incongruente), lo que permitía una comparación estricta de 
cada condición de control con su correspondiente condición experimental. La Figura 2 ilustra las diferentes combinaciones de las condiciones experimentales.

\section{Figura 2}

Secuencia de eventos en un ensayo e ilustración de las condiciones experimentales. Se muestra un ejemplo de estímulo facial en cada condición, donde 1 representa ojos de miedo + boca de enfado; 2: ojos de miedo + boca triste; 3 : ojos de miedo + boca alegre; 4,5 y 6: ojos de miedo + boca enmascarada; 7: ojos y boca de enfado; 8: ojos y boca tristes; 9: ojos y boca alegres; 10, 11 y 12: ojos de enfado, tristeza y alegría con boca enmascarada.

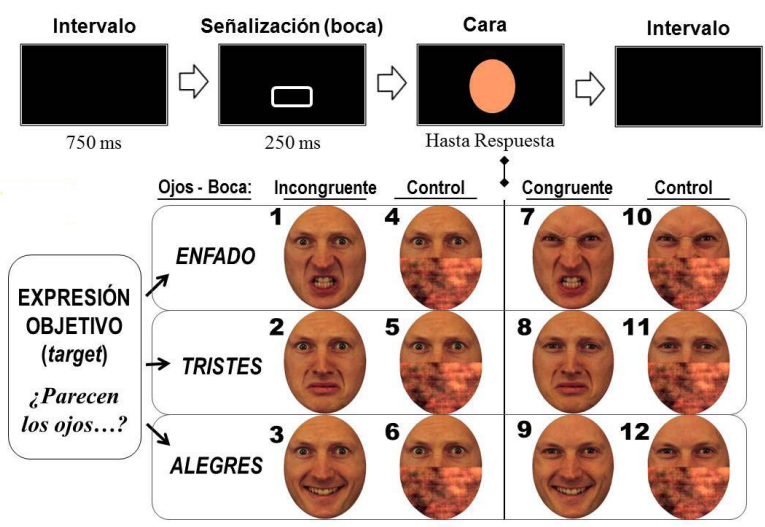

\section{Resultados}

\section{Congruencia Expresión Boca-Ojos}

Para analizar la influencia facilitadora en la percepción de la expresión de los ojos por parte de la boca con sonrisa, la de enfado y la triste, llevamos a cabo un ANOVA de 3 (Expresión Objetivo: alegre, enfado, triste) $\times 2$ (Boca: congruente vs. sin boca) sobre la probabilidad de responder que la expresión de los ojos correspondía a la expresión objetivo predefinida. Este

\section{Figura 3}

Probabilidad (en porcentaje) de responder que la expresión de los ojos se corresponde con la expresión objetivo, según sea ésta de "enfado", "tristeza" o "alegría"). * $p<.05 ; * * * p .001$ (diferencias significativas entre las condiciones experimentales con boca y las condiciones de control sin boca). Las letras a y b indican si la diferencia entre la condición experimental y la de control es mayor o menor (letras diferentes), o bien equivalente (misma letra o ausencia de letra), para un tipo de expresión objetivo que para otro.

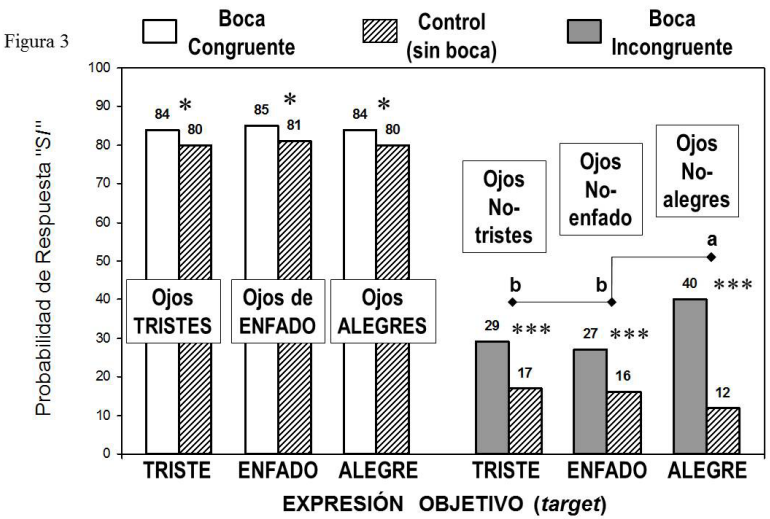

análisis se aplicó a las caras con expresión genuina y sus correspondientes caras de control. El análisis deparó únicamente un efecto principal de la boca, $F(1,35)=4.79, p<.05, \eta_{p}^{2}=.120$. El mismo tipo de ANOVA se llevó a cabo sobre los tiempos de reacción de las respuestas correctas. Igualmente, se produjo un efecto principal de la presencia de la boca, $F(1,35)=4.23, p$ $<.05, \eta_{p}{ }^{2}=.108$. Los resultados fueron convergentes en ambas medidas: En comparación con la ausencia de boca, la presencia de una boca congruente con los ojos facilitó la identificación de éstos - aumentando la probabilidad de los aciertos y disminuyendo su tiempo de reacción, siendo el efecto equivalente para todos los tipos de expresión objetivo. Véanse las Figuras 3 y 4.

\section{Incongruencia Expresión Boca-Ojos}

Para analizar la influencia interferidora de la boca con sonrisa, la de enfado y la triste sobre la valoración de la expresión de los ojos, aplicamos un ANOVA de 3 (Expresión Objetivo: alegre, enfado, triste) $\times 2$ (Boca: incongruente vs. sin boca) sobre la probabilidad de responder que la expresión de los ojos correspondía con la expresión objetivo predefinida. Este análisis se aplicó a las caras con expresión mixta y sus correspondientes caras de control. Apareció un efecto principal de la boca, $F(1$, $35)=84.08, p<.001, \eta_{p}^{2}=.706$, así como una interacción entre boca y expresión objetivo, $F(2,70)=11.10, p<.001, \eta_{p}^{2}=.241$. La comparación entre las condiciones con boca incongruente y sin boca para cada una de las expresiones objetivo mostró diferencias significativas tanto para la expresión alegre, $t(35)=$ 6.63, $p<.001, d=1.56, r=.62$ (39.4\% de la varianza), como la de enfado, $t(35)=5.17, p<.001, d=1.22, r=.52(27.1 \%$ de la varianza), como la triste, $t(35)=5.87, p<.001, d=1.38, r$ $=.57$ (32.4\% de la varianza). En todos los casos, la presencia de una boca incongruente aumentó la probabilidad de juzgar

\section{Figura 4}

Media de los tiempos de reacción (en milisegundos) de las respuestas correctas. ${ }^{*} p<.05 ;{ }^{* *} p<.01 ;{ }^{* * *} p<.001$ (diferencias significativas entre las condiciones experimentales con boca y las condiciones de control sin boca). Las letras a y b indican si la diferencia entre la condición experimental y la de control es mayor o menor (letras diferentes), o bien equivalente (misma letra o ausencia de letra), para un tipo de expresión objetivo que para otro.

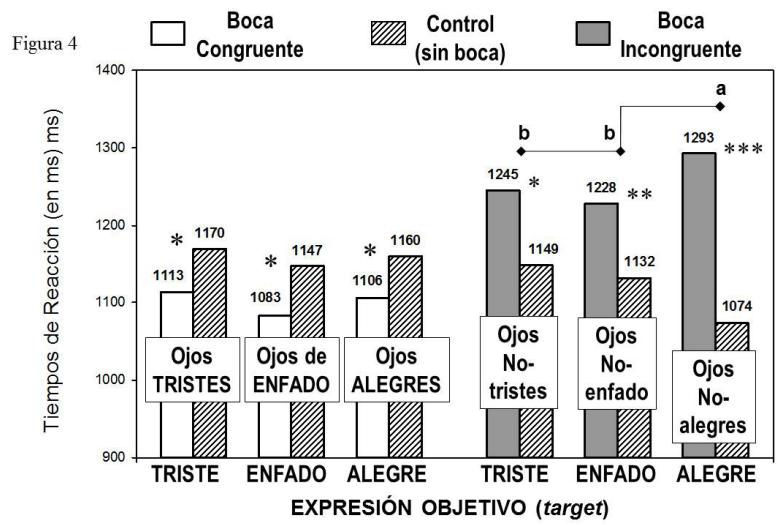


los ojos incorrectamente (como si se correspondieran con la expresión de la boca; v.g., juzgar ojos no alegres como alegres cuando aparecían en una boca con sonrisa). La interacción se debe a que las diferencias (incongruente vs. control) fueron mayores en el caso de la sonrisa ( $M$ probabilidad de responder "Si"= .27) que para la boca de enfado (.11) y la triste (.12). Así lo reveló un ANOVA de una vía (tipo de expresión objetivo) sobre las puntuaciones diferenciales (incongruente - control), $F(2,70)=11.10, p<.001, \eta_{p}^{2}=.241$, con sus correspondientes correcciones Bonferroni para comparaciones múltiples a posteriori $(p<.01)$. Así lo confirman, igualmente, los distintos tamaños del efecto referidos más arriba para cada contraste $t$. Esto demuestra un mayor efecto de la sonrisa que de la boca de enfado o la triste. Véanse las Figuras 3 y 4.

El mismo tipo de ANOVA (3: Expresión Objetivo) $\times 2$ (Boca) se llevó a cabo sobre los tiempos de reacción de las respuestas correctas. El efecto principal de la boca, $F(1,35)$ $=44.49, p<.001, \eta_{p}^{2}=.560$, fue modulado por la interacción entre boca y tipo de expresión objetivo, $F(2,70)=3.98, p<$ $.025, \eta_{p}{ }^{2}=.102$. La comparación entre las condiciones con boca incongruente y sin boca para cada una de las expresiones objetivo mostró diferencias significativas tanto para la alegre, $t(35)$ $=6.06, p<.001, d=1.43, r=.58$ (33.9\% de la varianza), como la de enfado, $t(35)=2.80, p<.01, d=0.66, r=.31(9.8 \%$ de la varianza), y la triste, $t(35)=2.63, p<.025, d=0.62, r=.30$ (8.8\% de la varianza). En todos los casos, la presencia de una boca incongruente con los ojos incrementó el tiempo necesario para responder correctamente que los ojos no se correspondían con la expresión objetivo. La interacción se debe a que las diferencias fueron mayores en el caso de la sonrisa $(219 \mathrm{~ms})$ que con la boca de enfado (96 ms) y la triste (95), como se reveló en un ANOVA de una vía (tipo de expresión objetivo) sobre las puntuaciones diferenciales (incongruente - control), $F(2,70)=$ $3.98, p<.025, \eta_{\mathrm{p}}{ }^{2}=.102$, y las correcciones Bonferroni para comparaciones múltiples $(p<.05)$. Así lo confirman, igualmente, los distintos tamaños del efecto referidos para cada contraste $t$. Esto corrobora el mayor efecto de la sonrisa. Véanse las Figuras 3 y 4.

\section{Discusión}

En la cara de una persona, la expresión de la boca afecta significativamente a la impresión que trasmiten los ojos al observador. En general, se produce una tendencia a juzgar los ojos en consonancia con la boca. Cuando la expresión de la boca es congruente con la de los ojos, las bocas de enfado y de tristeza producen efectos similares a la sonrisa, facilitando la identificación de la expresión de los ojos. La influencia de la expresión de la boca resulta especialmente notable cuando es incongruente con la de los ojos. En ese caso, la boca no sólo induce a juzgar erróneamente los ojos como si tuvieran la misma expresión que aquella, sino que también aumenta el tiempo para identificar correctamente la expresión de los ojos. En ese sentido, la boca "contamina" la expresión de los ojos, sesgando o distorsionando la impresión que éstos producen en el observador. Además, la boca con una sonrisa se ha revelado como más efectiva que la boca con expresión de enfado o tristeza cuando la boca es incongruente con los ojos. A continuación desarrollamos una explicación de estos fenómenos.

¿Por qué el mirar a una boca expresiva (sea alegre, triste, o de enfado) facilita el reconocimiento de una expresión de los ojos congruente con aquella, mientras que interfiere sobre el reconocimiento de una expresión no congruente? La explicación de este efecto puede establecerse en función del conocido mecanismo de priming o activación (v.g., Tulving y Schachter, 1990). Este mecanismo se ha propuesto para dar cuenta de la influencia que tiene el procesamiento de un estímulo que aparece primero sobre el procesamiento de otro posterior. Esencialmente, el sistema cognitivo inicia una representación perceptiva, semántica y afectiva de un estímulo desde el mismo momento de la aparición de éste, permaneciendo dicha representación activa o accesible en la memoria temporalmente. Dicha representación guía el análisis subsiguiente de otros elementos del mismo estímulo y de estímulos diferentes que aparecen próximos temporal o espacialmente. Esto implica que la representación inicial actúa como filtro y plantilla con los cuales interpretar la nueva información de modo acorde con la representación formada previamente. Existe considerable evidencia de dicho mecanismo en relación con el formato perceptivo de los estímulos (v.g., Mulligan y Hornstein, 2000), su contenido semántico (v.g., Ortells et al., 2001), y su valencia afectiva (v.g., Calvo y Nummenmaa, 2007).

Aplicando este mecanismo al reconocimiento de expresiones faciales, el procesamiento inicial de la boca predispondría al observador a juzgar otras partes del resto de la cara como si pertenecieran a la misma categoría expresiva. Si la boca a la que se mira primero es alegre (o triste o de enfado), se activará la correspondiente categoría. Esto, por un lado, facilitará la identificación de la expresión de los ojos a los que se mira a continuación, cuando coincide con la de la boca. Ahora bien, el sistema cognitivo del observador estará "esperando" una expresión de los ojos acorde con la que ha dejado activa la boca. Esto inducirá inicialmente a juzgar equivocadamente la expresión de los ojos cuando no coincida con la de la boca, inhibiendo o bloqueando la correcta identificación de aquella. De ahí la tendencia a evaluar de modo erróneo la expresión de los ojos, y a enlentecer su evaluación correcta, cuando los ojos no son congruentes con la boca. Es importante resaltar que, en nuestro estudio, la atención era dirigida (mediante una señal visual) inicialmente a región de la boca. En consecuencia, era la expresión de la boca la que proporcionaba la primera impresión de la cara, pudiendo así sesgar la evaluación de otras partes de ésta a las que se mirase a continuación. En general, estos datos y el mecanismo propuesto de priming son consistentes con la investigación que señala el poderoso influjo que tienen la primera mirada a la cara de una persona, a la hora de juzgar otros aspectos de ésta (Todorov, Pakrashi y Oostendorf, 2009), y cómo las 
primeras impresiones son muy resistentes al cambio incluso en presencia de información desconfirmatoria (Wyer, 2010).

¿Por qué la influencia de la boca sonriente es mayor que la de la triste o la de enfado? En principio, según lo recién argumentado, el mecanismo de priming sería común e igualmente válido para todas las expresiones. Efectivamente, así sucede cuando la expresión de la boca es congruente con la de los ojos, ya que en este caso la magnitud de los efectos de facilitación fueron equivalentes para las distintas categorías. ¿Cuál es, pues, la razón para que el mecanismo actúe con mayor intensidad o produzca mayores efectos de la sonrisa cuando la expresión no es congruente? La respuesta a esta pregunta requiere la consideración de dos propiedades especiales de la sonrisa, en las cuales destaca como rasgo facial con respecto a las otras expresiones emocionales (véase la introducción): la saliencia perceptiva (Calvo y Nummenmaa, 2008) y el valor diagnóstico (Calvo y Marrero, 2009). Por su mayor saliencia, la boca sonriente capta poderosamente la atención, compitiendo con ventaja por los recursos de procesamiento frente a otros rasgos faciales. Se puede pensar, no obstante, que esta ventaja tendría poca influencia en el presente contexto experimental, debido a que la atención ya era atraída artificialmente en primer lugar hacia la boca por una señal visual previa. Ahora bien, la saliencia no sólo juega un papel fundamental en atraer la primera fijación, sino que incluso puede proyectarse hacia los ojos mientras el observador los mira (Kontsevich y Tyler, 2004), permitiendo así a la sonrisa influir sobre los ojos incluso extrafovealmente (Calvo, Nummenmaa y Avero, 2010; Goren y Wilson, 2006).

Sin embargo, la mayor influencia de la sonrisa sobre los ojos no alegres probablemente se debe al valor diagnóstico o la distintividad de aquella. Es decir, la cualidad por la cual la boca sonriente es un rasgo exclusivo de las caras alegres. El reconocimiento de las otras expresiones emocionales depende de la boca (asco), o de los ojos (enfado y miedo), o de los ojos y la boca (tristeza y sorpresa), pero ninguna de ellas depende tanto de la boca como la expresión de alegría (Bassili, 1979; Calder et al., 2000; Calvo y Marrero, 2009; Kohler et al., 2004; Nusseck et al., 2008; Smith et al., 2005). En este marco cobra especial importancia el mecanismo de priming al que hemos recurrido para explicar el efecto general de la mirada inicial a la boca sobre la evaluación de los ojos. En el caso de la sonrisa, dado su valor diagnóstico, ésta crearía en el observador una representación inicial -y la consiguiente expectativa- de que se halla ante una cara alegre. De ahí los efectos subsiguientes de interferencia en el procesamiento de los ojos cuando éstos no son alegres y es, por tanto, necesario inhibir la representación inicial. Además, el alto valor diagnóstico de la sonrisa juega un papel esencial en la categorización de caras con expresión mixta (v.g., ojos de miedo/tristeza y boca sonriente, como en las sonrisas nerviosas; u ojos de asco/enfado y boca sonriente, como en las sonrisas sarcásticas). Así, según Fiorentini y Viviani (2009), los rasgos faciales aislados adquieren mayor relevancia en el reconocimiento de expresiones ambiguas. En estos casos, el juicio sobre la categoría expresiva de la cara se rige por la lógica de máxima verosimilitud. Es decir, qué rasgo es más probable encontrar en una expresión dada. La ambigüedad se resolvería en función del rasgo disponible que fuera más distintivo. Esto le otorga a la sonrisa una función decisiva en las caras con expresión incongruente.

En conclusión, mirar a una boca de enfado, alegría o tristeza facilita la posterior categorización expresiva de los ojos cuando existe congruencia emocional entre ambos (v.g., boca y ojos de enfado). Por el contrario, mirar previamente a una boca de enfado, tristeza y, muy especialmente, de alegría, interfiere sobre la posterior categorización expresiva de los ojos cuando existe incongruencia emocional entre ambos. El observador tiende a juzgar la expresión de los ojos en función de la expresión de la boca. Los mayores efectos de interferencia de la sonrisa se deben, probablemente, a su alta saliencia perceptiva y su carácter diagnóstico. Debido a ello, la sonrisa induciría a juzgar erróneamente los ojos no alegres como si fueran alegres y forzaría al observador a emplear más tiempo para categorizarlos correctamente como no alegres. Esto pone de relieve las dificultades a la hora de discriminar entre sonrisas auténticas y fingidas y, por ende, la identificación del estado emocional y las motivaciones de las personas con las que interactuamos. Esto tiene implicaciones prácticas importantes, al menos cuando no disponemos de otras fuentes de información segura sobre esas personas, como sucede a menudo en las relaciones sociales.

\section{Referencias}

1. Ambadar, Z., Cohn, J. F. y Reed, L. I. (2009). All smiles are not created equal: Morphology and timing of smiles perceived as amused, polite, and embarrassed/nervous. Journal of Nonverbal Behavior, 33, 17-34. http://dx.doi. org/10.1007/s10919-008-0059-5

2. Bassili, J. (1979). Emotion recognition: The role of facial movement and the relative importance of upper and lower areas of the face. Journal of Personality and Social Psychology, 37, 2049-2058. http://dx.doi.org/10.1037/00223514.37.11.2049

3. Calder, A. J., Young, A. W., Keane, J. y Dean, M. (2000). Configural information in facial expression perception. Journal of Experimental Psychology: Human Perception and Performance, 26, 527-551. http://dx.doi. org/10.1037/0096-1523.26.2.527

4. Calvo, M. G. y Lundqvist, D. (2008). Facial expressions of emotion (KDEF): Identification under different displayduration conditions. Behavior Research Methods, 40, 109115. http://dx.doi.org/10.3758/BRM.40.1.109

5. Calvo, M. G. y Marrero, H. (2009). Visual search of emotional faces: The role of affective content and featural distinctiveness. Cognition and Emotion, 23, 782-806. http:// dx.doi.org/10.1080/02699930802151654

6. Calvo, M. G. y Nummenmaa, L. (2007). Processing of unattended emotional visual scenes. Journal of Experi- 
mental Psychology: General, 136, 347-369. http://dx.doi. org/10.1037/0096-3445.136.3.347

7. Calvo, M. G. y Nummenmaa, L. (2008). Detection of emotional faces: Salient physical features guide effective visual search. Journal of Experimental Psychology: General, 137, 471-494. http://dx.doi.org/10.1037/a0012771

8. Calvo, M. G., Nummenmaa, L. y Avero, P. (2010). Recognition advantage of happy faces in extrafoveal vision: Featural and affective processing. Visual Cognition, 18, 1274-1297. http://dx.doi.org/10.1080/13506285.2010.481 $\underline{867}$

9. Ekman, P. y Friesen, W.V. (1978). Facial action coding system: A technique for the measurement of facial movement. Palo Alto, Calif.: Consulting Psychologists Press.

10. Eckstein, M. P., Shimozaki, S. S. y Abbey, C. K. (2002). The footprints of visual attention in the Posner cueing paradigm revealed by classification images. Journal of Vision, 2, 25-45. http://dx.doi.org/10.1167/2.1.3

11. Fiorentini, C. y Viviani, P. (2009). Perceiving facial expressions. Visual Cognition, 17, 373-411. http://dx.doi. org/10.1080/13506280701821019

12. Frischen, A., Eastwood, J. D. y Smilek, D. (2008). Visual search for faces with emotional expressions. Psychological Bulletin, 134, 662-676. http://dx.doi.org/10.1037/00332909.134.5.662

13. Goren, D. y Wilson, H. R. (2006). Quantifying facial expression recognition across viewing conditions. Vision Research, 46, 1253-1262. http://dx.doi.org/10.1016/j. visres.2005.10.028

14. Henderson, J. M., y Williams, C. C. y Falk, R. J. (2005). Eye movements are functional during face learning. Memory y Cognition, 33, 98-106. http://dx.doi.org/10.3758/ BF03195300

15. Itti, L. y Koch, C. (2000). A saliency-based search mechanism for overt and covert shifts of visual attention. Vision Research, 40, 1489-1506. http://dx.doi.org/10.1016/S00426989(99)00163-7

16. Johnston, L., Miles, L. y Macrae, C. (2010). Why are you smiling at me? Social functions of enjoyment and nonenjoyment smiles. British Journal of Social Psychology, 49, 107-127. http://dx.doi.org/10.1348/014466609X412476

17. Juth, P., Lundqvist, D., Karlsson, A. y Öhman, A. (2005). Looking for foes and friends: Perceptual and emotional factors when finding a face in the crowd. Emotion, 5, 379-395. http://dx.doi.org/10.1037/1528-3542.5.4.379

18. Kohler, C. G., Turner, T., Stolar, N. M., Bilker, W. B., Brensinger, C. M., Gur, R. E. y Gur, R. C. (2004). Differences in facial expressions of four universal emotions. Psychiatry Research, 128, 235-244. http://dx.doi.org/10.1016/j.psychres.2004.07.003

19. Kontsevich, L. L. y Tyler, C. (2004). What makes Mona Lisa smile? Vision Research, 44, 1493-1498. http://dx.doi. org/10.1016/j.visres.2003.11.027
20. Krumhuber, E. G. y Manstead, A. S. R. (2009). Can Duchenne smiles be feigned? New evidence on felt and false smiles. Emotion, 9, 807-820. http://dx.doi.org/10.1037/ a0017844

21. Leppänen, J. y Hietanen, J. K. (2004). Positive facial expressions are recognized faster than negative facial expressions, but why? Psychological Research, 69, 22-29. http://dx.doi.org/10.1007/s00426-003-0157-2

22. Leppänen, J. y Hietanen, J. K. (2007). Is there more in a happy face than just a big smile? Visual Cognition, 15, 468490. http://dx.doi.org/10.1080/13506280600765333

23. Leppänen, J., Tenhunen, M. y Hietanen, J. K. (2003). Faster choice-reaction times to positive than to negative facial expressions: The role of cognitive and motor processes. Journal of Psychophysiology, 17, 113-123. http://dx.doi. org/10.1027//0269-8803.17.3.113

24. Loughead, J., Gur, R. C., Elliott, M. y Gur, R. E. (2008). Neural circuitry for accurate identification of facial emotions. Brain Research, 1194, 37-44. http://dx.doi. org/10.1016/j.brainres.2007.10.105

25. Lundqvist, D., Flykt, A. y Öhman, A. (1998). The Karolinska Directed Emotional Faces - KDEF. CD-ROM from Department of Clinical Neuroscience, Psychology section, Karolinska Institutet, Stockholm, Sweden. ISBN 91-6307164-9

26. Milders, M., Sahraie, A. y Logan, S. (2008). Minimum presentation time for masked facial expression discrimination. Cognition and Emotion, 22, 63-82. http://dx.doi. org/10.1080/02699930701273849

27. Mulligan, N. W. y Hornstein, S. L. (2000). Attention and perceptual priming in the perceptual identification task. Journal of Experimental Psychology: Learning, Memory, and Cognition, 26, 626-637. http://dx.doi.org/10.1037/02787393.26.3.626

28. Niedenthal, P. M., Mermillod, M., Maringer, M. y Hess, U. (2010). The Simulation of Smiles (SIMS) model: Embodied simulation and the meaning of facial expression. Behavioral and Brain Sciences, 33, 417-433. http://dx.doi. org/10.1017/S0140525X10000865

29. Nusseck, M., Cunningham, D. V., Wallraven, C. y Bülthoff, H. H. (2008). The contribution of different facial regions to the recognition of conversational expressions. Journal of Vision, 8, 1, 1-23. http://dx.doi.org/10.1167/8.8.1

30. Oatley, K. y Jenkins, J. M. (1996). Understanding emotions. London: Blackwell.

31. Ortells, J. J., Abad, M. J., Noguera, C. y Lupiáñez, J. (2001). Influence of prime-probe onset asynchrony and prime precuing manipulations on semantic priming effects with words in a lexical-decision task. Journal of Experimental Psychology: Human Perception y Performance, 27, 75-91. http://dx.doi.org/10.1037/0096-1523.27.1.75

32. Palermo, R. y Coltheart, M. (2004). Photographs of facial expression: Accuracy, response times, and ratings of inten- 
sity. Behavior Research Methods, 36, 634-638. http:// dx.doi.org/10.3758/BF03206544

33. Remland, M. S., Jones, T. S. y Brinkman, H. (1995). Interpersonal distance, body orientation, and touch: Effects of culture, gender, and age. Journal of Social Psychology, 135, 281-297. http://dx.doi.org/10.1080/00224545.1995.9 713958

34. Richler, J. J., Mack, M. L., Gauthier, I. y Palmeri, T. J. (2009). Holistic processing of faces happens at a glance. Vision Research, 49, 2856-2861. http://dx.doi. org/10.1016/j.visres.2009.08.025

35. Smith, M. L., Cottrell, G., Gosselin, F. y Schyns, P. G. (2005). Transmitting an decoding facial expressions of emotions. Psychological Science, 16, 184-189. http:// dx.doi.org/10.1111/j.0956-7976.2005.00801.x

36. Tanaka, J. W., Kaiser, M., Butler, S. y Le Grand, R. (in press). Mixed emotions: Holistic and analytic perception of facial expressions. Cognition and Emotion.

37. Todorov, T., Pakrashi, M. y Oostendorf, N. N. (2009). Evaluating faces on trustworthiness after minimal exposure. Social Cognition, 27, 813-833. http://dx.doi.org/10.1521/ soco.2009.27.6.813

38. Tomkins, S.S. (1962). Affect, imagery, consciousness. Vol. 1: The positive affects. New York: Springer Publishing Co.

39. Torralba, A., Oliva, A., Castelhano, M. S. y Henderson, J. (2006). Contextual guidance of eye movements in real-world scenes: The role of global features in object search. Psychological Review, 113, 766-786. http://dx.doi. org/10.1037/0033-295X.113.4.766
40. Tottenham, N., Tanaka, J. W., Leon, A. C., McCarry, T., Nurse, M., Hare, T. A., Marcus, D.J., Westerlund, A., Casey, B.J. y Nelson, C. (2009). The NimStim set of facial expressions: Judgments from untrained research participants. Psychiatry Research, 168, 242-249. http://dx.doi. org/10.1016/i.psychres.2008.05.006

41. Tulving, E. y Schacter, D. L. (1990). Priming and human memory systems. Science, 247, 301-305. http://dx.doi. org/10.1126/science. 2296719

42. Walther, D. y Koch, C. (2006). Modelling attention to salient proto-objects. Neural Networks, 19, 1395-1407. http://dx.doi.org/10.1016/j.neunet.2006.10.001

43. Wyer, N. (2010). You never get a second chance to make a first (implicit) impression: The role of elaboration in the formation and revision of implicit impressions. Social Cognition, 28, 1-19. http://dx.doi.org/10.1521/soco.2010.28.1.1

Fecha de recepción: 14 de noviembre de 2011 Fecha de recepción de la versión modificada: 30 de enero de 2012 Fecha de aceptación: 2 de febrero de 2012 